\title{
Gagliardo-Nirenberg inequalities in weighted Orlicz spaces
}

\author{
by \\ AgnieszKa KaŁamajska and \\ Katarzyna Pietruska-PaŁuba (Warszawa)
}

\begin{abstract}
We derive inequalities of Gagliardo-Nirenberg type in weighted Orlicz spaces on $\mathbb{R}^{n}$, for maximal functions of derivatives and for the derivatives themselves. This is done by an application of pointwise interpolation inequalities obtained previously by the first author and of Muckenhoupt-Bloom-Kerman-type theorems for maximal functions.
\end{abstract}

1. Introduction. Interpolation inequalities for derivatives have been studied for a long time. Beginning with the pioneering works by Hadamard [19-21], Kneser [32], Landau [38-40], Hardy, Littlewood and Landau [22-24], Kolmogorov's celebrated article [34], and the famous results of Gagliardo [16] and Nirenberg [45], now there is a large body of literature on this subject (see e.g. [3, 7, 11, 12, 18, 25, 27, 36, 41-43, 46, 49] and their references).

Despite intensive investigation carried out in this area, there are only a few articles about interpolation inequalities in Orlicz spaces. The only ones we know of are contributions by Bang and coauthors [3-6] and our recent papers [26-28]. Here we continue the research in this direction.

Our main goal is to obtain inequalities of the form

$$
\int \Psi\left(\left|\nabla^{(k)} u\right|\right) d \mu \leq\left(\int \Psi_{1}(|u|) d \mu+\int \Psi_{2}\left(\left|\nabla^{(m)} u\right|\right) d \mu\right)
$$

and also

$$
\left\|\nabla^{(k)} u\right\|_{L^{\Psi}(\mu)} \leq c\|u\|_{L^{\Psi_{1}(\mu)}}^{1-k / m}\left\|\nabla^{(m)} u\right\|_{L^{\Psi_{2}(\mu)}}^{k / m},
$$

where $\Psi, \Psi_{1}$ and $\Psi_{2}$ are $\mathrm{N}$-functions satisfying certain consistency conditions, $0<k<m$ are positive integers, and $\mu$ belongs to some class of weighted Radon measures.

2000 Mathematics Subject Classification: Primary 26D10; Secondary 46E35, 46E30.

Key words and phrases: Gagliardo-Nirenberg inequalities, Orlicz spaces, maximal function.

This research was done while A.K. was visiting the Institute of Mathematics of the Polish Academy of Sciences, Warsaw. This author would like to thank IM PAN for hospitality.

The work of both authors is supported by a KBN grant no. 1-PO3A-008-29. 
Our results extend those of Bang [3] who dealt with inequalities (1.2) for one-variable function and the Lebesgue measure. In our previous papers [26-28], we have discussed inequalities (1.1), (1.2), together with their generalizations, for the Lebesgue measure. More precisely, in [26] and [28] we have obtained variants of (1.1) and (1.2) in various logarithmic Zygmund spaces (for $\mu=d x$ ) and our paper [27] was devoted to similar inequalities in general Orlicz spaces, but for the Lebesgue measure and $k=1, m=2$ only. They have arisen there as special cases of more general inequalities

$$
\int \Psi(|\nabla u|) d x \leq\left(\int \Psi_{1}\left(R_{1}\left(|u|,\left|\nabla^{(2)} u\right|\right)\right) d x+\int \Psi_{2}\left(R_{2}\left(|u|,\left|\nabla^{(2)} u\right|\right)\right) d x\right)
$$

and also

$$
\|\nabla u\|_{L^{\Psi}(\mu)} \leq C\left\|R_{1}\left(|u|,\left|\nabla^{(2)} u\right|\right)\right\|_{L^{\Psi_{1}(\mu)}}^{1 / 2}\left\|R_{2}\left(|u|,\left|\nabla^{(2)} u\right|\right)\right\|_{L^{\Psi_{2}(\mu)}}^{1 / 2},
$$

where $R_{1}, R_{2}:[0, \infty)^{2} \rightarrow \mathbb{R}_{+}$were continuous functions such that

$$
R_{1}\left(\lambda_{1}, \lambda_{2}\right) R_{2}\left(\lambda_{1}, \lambda_{2}\right)=\lambda_{1} \lambda_{2} .
$$

Note that inequalities (1.1) and (1.2) for $k=1, m=2$ and the Lebesgue measure correspond to $R_{1}\left(\lambda_{1}, \lambda_{2}\right)=\lambda_{1}$ and $R_{2}\left(\lambda_{1}, \lambda_{2}\right)=\lambda_{2}$. The results of [26-28] were based on the crucial observation that for a smooth function $u$ with bounded support the integral $\int \Psi(|\nabla u|) d x$ can be estimated by the quantity $\int N(|\nabla u|)|u|\left|\nabla^{(2)} u\right| d x$ (with a certain function $N$ ), which was then the object of further analysis.

Here we also deal with inequalities in the form (1.1) and (1.2), but our present approach is essentially different and brings new results. Namely, our starting point is the following pointwise multiplicative inequality expressed in terms of the Hardy-Littlewood maximal functions, obtained by the first author in [25]:

$$
\mathcal{M}\left(\nabla^{(k)} f\right)(x) \leq C_{0}(\mathcal{M} f(x))^{1-k / m}\left(\mathcal{M}\left(\nabla^{(m)} f\right)(x)\right)^{k / m},
$$

holding true for every $f \in W_{\text {loc }}^{m, 1}\left(\mathbb{R}^{n}\right)$ and almost all $x \in \mathbb{R}^{n}$. Then, using Young inequalities in Orlicz spaces, we obtain counterparts of (1.1) and (1.2) for maximal functions (see Theorem 3.1):

$$
\int_{\mathbb{R}^{n}} \Phi\left(C s \mathcal{M}\left(\nabla^{(k)} f\right)\right) d \mu \leq \int_{\mathbb{R}^{n}} \Phi_{1}\left(s_{1} \mathcal{M} f\right) d \mu+\int_{\mathbb{R}^{n}} \Phi_{2}\left(s_{2} \mathcal{M}\left(\nabla^{(m)} f\right)\right) d \mu
$$

and

$$
\left\|\mathcal{M}\left(\nabla^{(k)} f\right)\right\|_{L^{\Phi}(\mu)} \leq C_{1}\|\mathcal{M} f\|_{L^{\Phi_{1}(\mu)}}^{1-k / m}\left\|\mathcal{M}\left(\nabla^{(m)} f\right)\right\|_{L^{\Phi_{2}(\mu)}}^{k / m},
$$

where $\Phi, \Phi_{1}, \Phi_{2}$ are certain functions, $\mu$ is an arbitrary nonnegative Radon measure, and $s, s_{1}, s_{2}$ are certain positive numbers. Inequality (1.6) requires $\Phi, \Phi_{1}, \Phi_{2}$ to be $\mathrm{N}$-functions, but (1.5) is valid for some nonconvex functions as well. In Section 4 inequalities (1.5) and (1.6) are then transformed into 
(1.1) and (1.2). This is done by an application of various MuckenhouptBloom-Kerman-type direct and reverse inequalities for maximal functions from $[8,30,31]$, within appropriate classes of measures.

Although inequalities (1.3) and (1.4) do not follow from our new methods, those derived now extend some of the previously obtained ones (for $\mu=d x, k=1, m=2$ ) to wider classes of admissible functions and measures. More precisely, when we restrict ourselves to $k=1, m=2, \mu=d x$, then the Gagliardo-Nirenberg inequalities (1.1) and (1.2) are a special case of (1.3) and (1.4). These are the inequalities that are generalized presently. See Section 5 for a detailed discussion.

We hope that the results presented here will contribute to the development of regularity theory for PDE's in Orlicz spaces, similarly to the classical case. For various regularity results in Orlicz-Sobolev spaces and motivations we refer to the papers $[10,14,47,51]$ and their references.

Acknowledgements. The authors would like to thank Professors: Andrea Cianchi, Amiran Gogatishvili, Miroslav Krbec and Luboš Pick for discussions.

2. Preliminaries and notation. We start by recalling preliminary facts about Orlicz spaces, referring e.g. to [35] for details.

Suppose that $\mu$ is a positive Radon measure on $\mathbb{R}^{n}$ and let $\Phi$ : $[0, \infty) \rightarrow[0, \infty)$ be an $N$-function, i.e. a strictly convex function satisfying $\lim _{\lambda \rightarrow 0} \Phi(\lambda) / \lambda=0$ and $\lim _{\lambda \rightarrow \infty} \Phi(\lambda) / \lambda=\infty$.

The weighted Orlicz space $L^{\Phi}(\mu)$ is the space

$$
L^{\Phi}(\mu):=\left\{f \text { measurable }: \int_{\mathbb{R}^{n}} \Phi(|f(x)| / K) d \mu(x) \leq 1 \text { for some } K>0\right\},
$$

equipped with the Luxemburg norm

$$
\|f\|_{L^{\Phi}(\mu)}=\inf \left\{K>0: \int_{\mathbb{R}^{n}} \Phi(|f(x)| / K) d \mu(x) \leq 1\right\} .
$$

This norm is complete and turns $L^{\Phi}(\mu)$ into a Banach space. When $\mu$ is the Lebesgue measure, it is dropped from the notation. For $\Phi(\lambda)=\lambda^{p}$ with $p>1$, the space $L^{\Phi}(\mu)$ coincides with the usual $L^{p}(\mu)$ space.

The symbol $\Phi^{*}$ denotes the Legendre transform of an $\mathrm{N}$-function $\Phi$, i.e. $\Phi^{*}(y)=\sup _{x>0}[x y-\Phi(x)]$, defined for $y \geq 0$. It is again an N-function and from its definition we get the Young inequality:

$$
x y \leq \Phi(x)+\Phi^{*}(y) \quad \text { for } x, y \geq 0 .
$$

$\Phi$ is said to satisfy the $\Delta_{2}$-condition if, for some constant $c>0$ and every $\lambda>0$, we have

$$
\Phi(2 \lambda) \leq c \Phi(\lambda) .
$$


In the class of differentiable convex functions the $\Delta_{2}$-condition is equivalent to

$$
\lambda \Phi^{\prime}(\lambda) \leq c \Phi(\lambda)
$$

satisfied for every $\lambda>0$, with a constant $c$ independent of $\lambda$ (see e.g. [35, Theorem 4.1]).

We will need the following two properties of modular functionals (see [35, formulas (9.20) and (9.4)]): for every $f \in L^{\Phi}(\mu)$ we have

$$
\|f\|_{L^{\Phi}(\mu)} \leq \int_{\mathbb{R}^{n}} \Phi(|f(x)|) d \mu(x)+1
$$

and also ([35, formula (9.21)])

$$
\int_{\mathbb{R}^{n}} \Phi\left(\frac{f(x)}{\|f\|_{L^{\Phi}(\mu)}}\right) d \mu(x) \leq 1 .
$$

If $\Phi$ satisfies the $\Delta_{2}$-condition, then (2.5) becomes an equality.

The function $\Phi_{1}$ is said to dominate $\Phi_{2}$ (symbolically: $\Phi_{2} \prec \Phi_{1}$ ) if there exist two positive constants $K_{1}, K_{2}$ such that $\Phi_{2}(\lambda) \leq K_{1} \Phi_{1}\left(K_{2} \lambda\right)$ for every $\lambda>0$. In that case we have

$$
\|\cdot\|_{L_{2}^{\Phi}(\mu)} \leq K\|\cdot\|_{L_{1}^{\Phi}(\mu)} \quad \text { with } K=K_{2}\left(K_{1}+1\right) .
$$

Functions $\Phi_{1}$ and $\Phi_{2}$ are called equivalent (symbolically $\Phi_{1} \asymp \Phi_{2}$ ) when $\Phi_{2} \prec \Phi_{1}$ and $\Phi_{1} \prec \Phi_{2}$. In particular equivalent $\mathrm{N}$-functions give rise to equivalent Luxemburg norms.

We use the standard notation: $C_{0}^{k}\left(\mathbb{R}^{n}\right)$ for compactly supported functions of class $C^{k}$ defined on $\mathbb{R}^{n}$, and $L^{p}\left(\mathbb{R}^{n}\right), L_{\text {loc }}^{p}\left(\mathbb{R}^{n}\right), W^{k, p}\left(\mathbb{R}^{n}\right), W_{\text {loc }}^{k, p}\left(\mathbb{R}^{n}\right)$ for the $L^{p}$ and Sobolev spaces respectively. By $\widetilde{C}_{0}\left(\mathbb{R}^{n}\right)$ we denote the continuous functions on $\mathbb{R}^{n}$ vanishing at infinity, while $L^{0, \Phi}(\mu)$ stands for the completion of $\widetilde{C}_{0}\left(\mathbb{R}^{n}\right)$ in the space $L^{\Phi}(\mu)$. By $\nabla^{(k)} f$ we denote the vector $\left(D^{\alpha} f\right)_{|\alpha|=k}$, understood in the classical sense (we assume that $f$ is of class $C^{k}$ ). If $w$ is a vector in Euclidean space then $|w|$ stands for its standard norm.

The letter $c$ is reserved to denote a generic constant, whose value can change from line to line. The relevant constants are denoted by upper-case letters.

3. Inequalities involving maximal functions. Let $f \in L_{\mathrm{loc}}^{1}\left(\mathbb{R}^{n}\right)$. The Hardy-Littlewood maximal function of $f$ is defined as

$$
\mathcal{M} f(x)=\sup _{S: x \in S} \frac{1}{|S|} \int_{S}|f(y)| d y,
$$

where the supremum is taken over all cubes $S$ in $\mathbb{R}^{n}$ containing $x$ with edges parallel to the axes, and $|S|$ denotes their Lebesgue measure (see e.g. [33, 50]). 
For a vector-valued function $f$, the symbol $\mathcal{M} f$ stands for the maximal function of $|f|$.

The main result of this section reads as follows.

TheOREM 3.1. Let $k, m \in \mathbb{Z}_{+}$with $0<k<m$. Suppose that $\mu$ is an arbitrary positive Radon measure on $\mathbb{R}^{n}, \Phi:[0, \infty) \rightarrow[0, \infty)$ is a nondecreasing function, and $F:[0, \infty) \rightarrow[0, \infty)$ an $N$-function. Set

$$
\Phi_{1}(\lambda)=\Phi\left(F\left(\lambda^{1-k / m}\right)\right), \quad \Phi_{2}(\lambda)=\Phi\left(F^{*}\left(\lambda^{k / m}\right)\right) .
$$

Then there exists a constant $C=C(n)>0$ such that for every $f \in C_{0}^{m}\left(\mathbb{R}^{n}\right)$ and any numbers $s, s_{1}, s_{2}>0$ which satisfy $s=s_{1}^{1-k / m} s_{2}^{k / m}$ one has

$$
\int_{\mathbb{R}^{n}} \Phi\left(C s \mathcal{M}\left(\nabla^{(k)} f\right)\right) d \mu \leq \int_{\mathbb{R}^{n}} \Phi_{1}\left(s_{1} \mathcal{M} f\right) d \mu+\int_{\mathbb{R}^{n}} \Phi_{2}\left(s_{2} \mathcal{M}\left(\nabla^{(m)} f\right)\right) d \mu .
$$

Moreover, if $\Phi, \Phi_{1}$ and $\Phi_{2}$ are $N$-functions, then also

$$
\left\|\mathcal{M}\left(\nabla^{(k)} f\right)\right\|_{L^{\Phi}(\mu)} \leq C_{1}\|\mathcal{M} f\|_{L^{\Phi_{1}(\mu)}}^{1-k / m}\left\|\mathcal{M}\left(\nabla^{(m)} f\right)\right\|_{L^{\Phi_{2}(\mu)}}^{k / m},
$$

with a constant $C_{1}$ independent of $f \in C_{0}^{m}\left(\mathbb{R}^{n}\right)$.

Proof. Our result is based on the following version of the GagliardoNirenberg inequality obtained by the first author in [25] (see also [41, 42] for other related inequalities):

$$
\mathcal{M}\left(\nabla^{(k)} f\right)(x) \leq C_{0}(\mathcal{M} f(x))^{1-k / m}\left(\mathcal{M}\left(\nabla^{(m)} f\right)(x)\right)^{k / m},
$$

holding for every $f \in W_{\mathrm{loc}}^{m, 1}\left(\mathbb{R}^{n}\right)$ and almost every $x$ (with respect to the Lebesgue measure), with a constant $C_{0}>0$ depending on dimension only.

It is not hard to show that for $f \in C_{0}\left(\mathbb{R}^{n}\right)$ one has $\mathcal{M} f \in \widetilde{C}_{0}\left(\mathbb{R}^{n}\right)$. In particular, for $f \in C_{0}^{m}\left(\mathbb{R}^{n}\right)$ all the functions

$$
g(x)=\frac{1}{2 C_{0}} \mathcal{M}\left(\nabla^{(k)} f\right)(x), \quad g_{1}(x)=\mathcal{M} f(x), \quad g_{2}(x)=\mathcal{M}\left(\nabla^{(m)} f\right)(x)
$$

belong to $\widetilde{C}_{0}\left(\mathbb{R}^{n}\right)$, so that the inequality (3.4) holds true for every $x \in \mathbb{R}^{n}$.

Let $s_{1}, s_{2}$ be arbitrary positive numbers, $\alpha_{1}=1-k / m, \alpha_{2}=k / m$, $s=s_{1}^{\alpha_{1}} s_{2}^{\alpha_{2}}, \widetilde{g}=s g, \widetilde{g}_{1}=s_{1} g_{1}, \widetilde{g}_{2}=s_{2} g_{2}$. Then (3.4) can be rewritten as

$$
\widetilde{g}(x) \leq \frac{1}{2} \widetilde{g}_{1}(x)^{\alpha_{1}} \widetilde{g}_{2}(x)^{\alpha_{2}} .
$$

Since $\Phi$ is monotone, (3.4) combined with the Young inequality (2.1) implies that

$$
\begin{aligned}
\Phi(\widetilde{g}(x)) & \leq \Phi\left(\frac{1}{2}\left(F\left(\widetilde{g}_{1}(x)^{\alpha_{1}}\right)+F^{*}\left(\widetilde{g}_{2}(x)^{\alpha_{2}}\right)\right)\right) \\
& \leq \Phi\left(\max \left\{F\left(\widetilde{g}_{1}(x)^{\alpha_{1}}\right), F^{*}\left(\widetilde{g}_{2}(x)^{\alpha_{2}}\right)\right\}\right) \\
& \leq \Phi_{1}\left(\widetilde{g}_{1}(x)\right)+\Phi_{2}\left(\widetilde{g}_{2}(x)\right) .
\end{aligned}
$$


Integrating this over $\mathbb{R}^{n}$ with respect to $\mu$ gives

$$
\begin{aligned}
\int_{\mathbb{R}^{n}} \Phi\left(\frac{s}{2 C_{0}} \mathcal{M}\left(\nabla^{(k)} f\right)\right) d \mu \\
\quad \leq \int_{\mathbb{R}^{n}} \Phi_{1}\left(s_{1} \mathcal{M} f\right) d \mu+\int_{\mathbb{R}^{n}} \Phi_{2}\left(s_{2} \mathcal{M}\left(\nabla^{(m)} f\right)\right) d \mu .
\end{aligned}
$$

For $C=\left(2 C_{0}\right)^{-1}$, this is $(3.2)$.

To prove (3.3), assume that both $\|\mathcal{M} f\|_{L^{\Phi_{1}(\mu)}}$ and $\left\|\mathcal{M}\left(\nabla^{(m)} f\right)\right\|_{L^{\Phi_{2}(\mu)}}$ are nonzero. This can be done without loss of generality, as otherwise $f \equiv 0$. Then take $s_{1}=\left(\|\mathcal{M} f\|_{L^{\Phi_{1}(\mu)}}\right)^{-1}$ and $s_{2}=\left(\left\|\mathcal{M}\left(\nabla^{(m)} f\right)\right\|_{L^{\Phi_{2}(\mu)}}\right)^{-1}$. Using (2.5), from (3.7) we obtain $\int \Phi\left(\frac{s}{2 C_{0}} \mathcal{M}\left(\nabla^{(k)} f\right)\right) d \mu \leq 2$, which further gives that $\int \Phi_{0}\left(\frac{s}{2 C_{0}} \mathcal{M}\left(\nabla^{(k)} f\right)\right) d \mu \leq 1$, where we put $\Phi_{0}(\lambda)=\frac{1}{2} \Phi(\lambda)$. Consequently, from the very definition of the Luxemburg norm,

$$
\left\|\mathcal{M}\left(\nabla^{(k)} f\right)\right\|_{L^{\Phi_{0}}(\mu)} \leq 2 C_{0} s^{-1}=2 C_{0} s_{1}^{-\alpha_{1}} s_{2}^{-\alpha_{2}} .
$$

As equivalent $\mathrm{N}$-functions give rise to equivalent norms, this gives (3.3). More precisely, one applies (2.6) to get

$$
\left\|\mathcal{M}\left(\nabla^{(k)} f\right)\right\|_{L^{\Phi}(\mu)} \leq 3\left\|\mathcal{M}\left(\nabla^{(k)} f\right)\right\|_{L^{\Phi_{0}}(\mu)},
$$

which together with (3.8) implies (3.3) with $C_{1}=6 C_{0}$.

The $\Delta_{2}$-condition permits to move the constant $C$ in (3.2) outside the integral. In this case we have the following result.

Corollary 3.1. Let $k, m \in \mathbb{Z}$ with $0<k<m$. Suppose that $\mu$ is an arbitrary nonnegative Radon measure on $\mathbb{R}^{n}, F:[0, \infty) \rightarrow[0, \infty)$ is an $N$-function, $\Phi:[0, \infty) \rightarrow[0, \infty)$ is a nondecreasing function, $\Phi_{1}$ and $\Phi_{2}$ are given by (3.1), and additionally at least one of the functions $\Phi, \Phi_{1}, \Phi_{2}$ satisfies the $\Delta_{2}$-condition. Then there exists a constant $\widetilde{C}>0$ such that for every $f \in C_{0}^{m}\left(\mathbb{R}^{n}\right)$ one has

$$
\int_{\mathbb{R}^{n}} \Phi\left(\mathcal{M}\left(\nabla^{(k)} f\right)\right) d \mu \leq \widetilde{C}\left(\int_{\mathbb{R}^{n}} \Phi_{1}(\mathcal{M} f) d \mu+\int_{\mathbb{R}^{n}} \Phi_{2}\left(\mathcal{M}\left(\nabla^{(m)} f\right)\right) d \mu\right) .
$$

Proof. If $\Phi$ satisfies the $\Delta_{2}$-condition, then we apply (3.7) with $s=s_{1}=$ $s_{2}=1$ and then the $\Delta_{2}$-condition. On the other hand, when one of the functions $\Phi_{1}, \Phi_{2}$, say $\Phi_{1}$, satisfies the $\Delta_{2}$-condition, one applies (3.7) for $s_{1}=\left(2 C_{0}\right)^{m /(m-k)}, s_{2}=1, s=2 C_{0}$. To conclude, one uses the $\Delta_{2}$-condition (2.2) for the function $\Phi_{1}$.

REMARK 3.1. Note that the Radon measure admissible in inequalities (3.2), (3.3), and (3.9) can be taken arbitrary. In particular it need not be absolutely continuous with respect to the Lebesgue measure. Also, we do not 
require the function $\Phi$ to be convex in order for (3.2) and (3.9) to hold. Its monotonicity is sufficient.

REMARK 3.2. We already know from the proof of Theorem 3.1 that (3.4) implies (3.2). On the other hand, if (3.2) is satisfied for an arbitrary Radon measure $\mu$ and arbitrary $\Phi, F, s, s_{1}, s_{2}$ as in the statement of Theorem 3.1, then (3.2) implies (3.4). Indeed, once we take $\mu=\delta_{\{x\}}$ to be the Dirac measure, $\Phi=\mathrm{id}, s=s_{1}=s_{2}=1$ and $F(\lambda)=\lambda^{m /(m-k)}$, we arrive at

$$
\mathcal{M}\left(\nabla^{(k)} f\right)(x) \leq C\left(\mathcal{M} f(x)+\mathcal{M}\left(\nabla^{(m)} f\right)(x)\right) .
$$

Then (3.4) is obtained by the classical rescaling argument: first substitute $f_{t}(x)=f(t x)$ in the inequality above and then optimize with respect to $t>0$.

REMARK 3.3. An obvious corollary of (3.4) is the following inequality, holding for an arbitrary nondecreasing function $\Phi$ and $f \in C_{0}^{m}\left(\mathbb{R}^{n}\right)$ :

$$
\left.\int_{\mathbb{R}^{n}} \Phi\left(\mathcal{M}\left(\nabla^{(k)} f\right)\right) d \mu \leq \int_{\mathbb{R}^{n}} \Phi\left(C_{0}(\mathcal{M} f)^{1-k / m}\left(\mathcal{M}\left(\nabla^{(m)} f\right)\right)^{k / m}\right)\right) d \mu
$$

where $\mu$ is an arbitrary Radon measure, $k, m \in \mathbb{N}, 0<k<m$, and the constant $C_{0}$ is independent of $f$. In [26] we have shown that if $\Phi$ satisfies the "differential" $\Delta_{2}$-condition (2.3) (but is not necessarily convex) and moreover $\Phi(\lambda) / \lambda^{2}$ is nondecreasing, then for every $f \in C_{0}^{\infty}\left(\mathbb{R}^{n}\right)$ we have

$$
\int_{\mathbb{R}^{n}} \Phi(|\nabla f|) d x \leq \int_{\mathbb{R}^{n}} \Phi\left(C \sqrt{|f|\left|\nabla^{(2)} f\right|}\right) d x .
$$

Obviously, as $|h| \leq \mathcal{M} h$ almost everywhere, we see that both inequalities (3.10) (considered for $\mu=d x, k=1, m=2$ ) and (3.11) imply

$$
\int_{\mathbb{R}^{n}} \Phi(|\nabla f|) d x \leq \int_{\mathbb{R}^{n}} \Phi\left(C \sqrt{\mathcal{M} f \cdot \mathcal{M}\left(\nabla^{(2)} f\right)}\right) d x
$$

but (3.10) (for $\mu=d x, k=1, m=2$ ) and (3.11) do not seem to be equivalent.

4. Inequalities for derivatives. We now aim at transforming inequalities (3.2) and (3.3) into corresponding inequalities of Gagliardo-Nirenberg type involving derivatives rather than maximal functions.

As a tool we present below some strong type inequalities for the HardyLittlewood maximal operator between Orlicz spaces. The results summarized in Section 4.1 are known.

4.1. Strong-type inequalities for maximal functions. Summary of known results. In this section we deal with positive absolutely continuous Radon measures on $\mathbb{R}^{n}, \mu(d x)=w(x) d x$, where $w(x)$ is a weight function (a nonnegative, locally integrable function $\left.w: \mathbb{R}^{n} \rightarrow[0, \infty)\right)$ satisfying certain conditions described below. 
The following definition and the subsequent theorem are due to Bloom and Kerman (see [8]).

Definition 4.1 (the class $W_{\Phi}$ ). Suppose that $\Phi:[0, \infty) \rightarrow[0, \infty)$ is an $\mathrm{N}$-function. We say that a weight $w: \mathbb{R}^{n} \rightarrow[0, \infty)$ of a measure $\mu(d x)=$ $w(x) d x$ belongs to the class $W_{\Phi}$ if for all cubes $S \subset \mathbb{R}^{n}$ and all $\lambda>0$,

$$
\int_{S} \Phi^{*}\left(\frac{\Phi(\lambda) \mu(S)}{c \lambda|S| w(x)}\right) w(x) d x \leq \Phi(\lambda) \mu(S)<\infty,
$$

with a constant $c>0$ independent of $S$.

If $\Phi(\lambda)=\lambda^{p}, p>1$, then the class $W_{\Phi}$ coincides with the class of $A_{p^{-}}$ weights (see e.g. [50] for the definition).

THEOREM 4.1. Suppose that $\Phi$ is an $N$-function and let $w$ be a nonnegative weight on $\mathbb{R}^{n}$. Then the following two statements are equivalent:

(a) For all measurable u,

$$
\int_{\mathbb{R}^{n}} \Phi(\mathcal{M} u(x)) w(x) d x \leq \int_{\mathbb{R}^{n}} \Phi(B|u(x)|) w(x) d x,
$$

with some constant $B=B_{\Phi}$ not depending on $u$.

(b) $\Phi^{*}$ satisfies the $\Delta_{2}$-condition and $w \in W_{\Phi}$.

Following [8], if $\Phi^{*}$ satisfies the $\Delta_{2}$-condition, then we write $\Phi \in \Delta_{2}^{\mathrm{c}}$.

This theorem completely describes the class of weights for which the inequality (4.1) holds. In particular, if $\Phi^{*}$ does not satisfy the $\Delta_{2}$-condition, then there is no weight $w$ for which (4.1) could possibly hold for all measurable functions $u$. Theorem 4.1 generalizes the Muckenhoupt theorem (see e.g. [44]), which asserts that the maximal operator is of strong type $(p, p)$ if and only if the weight considered is an $A_{p}$-weight. After Muckenhoupt, variants of Theorem 4.1 were addressed also by Kerman and Torchinsky under the assumption that both $\Phi$ and $\Phi^{*}$ satisfy the $\Delta_{2}$-condition (see [29]), Kokilashvili and Krbec (Theorem 8.3.1 on page 339 in [33]) and Lai [37]. See also the references in those papers.

REMARK 4.1. If $\Phi_{1} \asymp \Phi_{2}$, then also $\Phi_{1}^{*} \asymp \Phi_{2}^{*}$, and so the $\Delta_{2}^{\mathrm{c}}$-property is shared by all equivalent functions. It is also easy to see that if $\Phi_{1} \asymp \Phi_{2}$ and $\Phi_{1} \in \Delta_{2}^{\mathrm{c}}$, then $W_{\Phi_{1}}=W_{\Phi_{2}}$. Indeed, suppose that $\Phi_{1} \asymp \Phi_{2}$ and $w \in W_{\Phi_{1}}$. Then according to Theorem 4.1 we have

$$
\int_{\mathbb{R}^{n}} \Phi_{1}(\mathcal{M} u(x)) w(x) d x \leq \int_{\mathbb{R}^{n}} \Phi_{1}(B u(x)) w(x) d x,
$$

with a constant $B$ independent of $u$. This implies

$$
\int_{\mathbb{R}^{n}} \Phi_{2}\left(C_{1} \mathcal{M} u(x)\right) w(x) d x \leq C_{2} \int_{\mathbb{R}^{n}} \Phi_{2}\left(C_{3} u(x)\right) w(x) d x,
$$


with constants $C_{1}, C_{2}, C_{3}$ independent of $u$. Substitution $\widetilde{u}=C_{1} u$ leads to a similar inequality with $C_{1}=1$ (and possibly different $C_{3}$ ). We may also assume that $C_{2}>1$. Next, the convexity of $\Phi_{2}$ together with the property $\Phi_{2}(0)=0$ give $C_{2} \Phi_{2}(\lambda) \leq \Phi_{2}\left(C_{2} \lambda\right)$, which implies

$$
\int_{\mathbb{R}^{n}} \Phi_{2}(\mathcal{M} u(x)) w(x) d x \leq \int_{\mathbb{R}^{n}} \Phi_{2}(C u(x)) w(x) d x,
$$

with a constant $C$ independent of $u$. Finally, again from Theorem 4.1, we get $w \in W_{\Phi_{2}}$ (and $\Phi_{2} \in \Delta_{2}^{\mathrm{c}}$ ), which is exactly what we claimed.

As it is usually not possible to express $\Phi^{*}$ in a closed form, below we present a quantitative tool which permits one to decide whether a given function belongs to the class $\Delta_{2}^{\mathrm{c}}$. It is basically taken from [35, Theorem 4.3]. Since slight modifications are needed, the proof is given in the Appendix.

Proposition 4.1. Suppose that $\Phi:[0, \infty) \rightarrow[0, \infty)$ is a continuously differentiable $N$-function. Then the following three statements are equivalent:

(D1) $\Phi \in \Delta_{2}^{\mathrm{c}}$,

(D2) there exists a constant $\alpha>1$ such that for all $t>0$ we have $t \Phi^{\prime}(t) / \Phi(t) \geq \alpha$

(D3) there exists a constant $\alpha>1$ such that $\Phi(t) / t^{\alpha}$ is nondecreasing.

Results similar to Theorem 4.1 are also available when $\Phi^{*}$ does not satisfy the $\Delta_{2}$-condition. In this case one has to deal with two distinct Orlicz spaces $L_{\Phi}$ and $L_{\Psi}$. For related results we refer to [2, 8, 13, 17, 29-31, 33, 37, 48].

Below (Theorem 4.2) we present two results which were included in Kita's papers $[30,31]$. For completeness, also the proof of Theorem 4.2 is given in the Appendix.

To continue, we recall two further classes of weights.

Definition 4.2. We say that a weight function $w: \mathbb{R}^{n} \rightarrow[0, \infty)$ belongs to the $A_{1}$-class $\left(w \in A_{1}\right)$ if there exists a constant $C>0$ such that for every open cube $S \subseteq \mathbb{R}^{n}$ we have

$$
\frac{1}{|S|} \int_{S} w(y) d y \leq C \underset{x \in S}{\operatorname{essinf}} w(x) .
$$

Definition 4.3. We say that a weight function $w: \mathbb{R}^{n} \rightarrow[0, \infty)$ belongs to the $A_{\infty}^{\prime}$-class $\left(w \in A_{\infty}^{\prime}\right)$ if there exists a constant $C>0$ such that for every open cube $S \subseteq \mathbb{R}^{n}$ we have

$$
\frac{1}{|S|} \int_{2 S} w(y) d y \geq C \underset{x \in S}{\operatorname{ess} \sup } w(x) .
$$


Following Kita, for a weight function $w: \mathbb{R}^{n} \rightarrow[0, \infty)$, we write

$$
\mathcal{R}_{0}(w)=\left\{f \text { measurable: } \int_{\{|f|>t\}}|f(x)| w(x) d x<\infty \text { for every } t>0\right\} .
$$

TheOREM 4.2. Suppose that $a, b:[0, \infty) \rightarrow[0, \infty)$ are functions such that $a(s) / s$ is locally integrable on $(0, \infty)$,

$$
\int_{0}^{1} \frac{a(t)}{t} d t<\infty \quad \text { and } \quad b(t)=\int_{0}^{t} \frac{a(s)}{s} d s .
$$

Set

$$
\Phi(t)=\int_{0}^{t} a(s) d s \quad \text { and } \quad \Psi(t)=\int_{0}^{t} b(s) d s .
$$

Then the following two statements hold true:

1. If $w \in A_{1}$, then for every $f \in L_{\mathrm{loc}}^{1} \cap \mathcal{R}_{0}(w)$,

$$
\int_{\mathbb{R}^{n}} \Phi(\mathcal{M} f(x)) w(x) d x \leq K_{1} \int_{\mathbb{R}^{n}} \Psi(|f(x)|) w(x) d x .
$$

2. If $w \in A_{\infty}^{\prime}$, then for every $f \in L_{\mathrm{loc}}^{1} \cap \mathcal{R}_{0}(w)$,

$$
\int_{\mathbb{R}^{n}} \Psi(|f(x)|) w(x) d x \leq K_{2} \int_{\mathbb{R}^{n}} \Phi(\mathcal{M} f(x)) w(x) d x .
$$

The constants $K_{1}, K_{2}>0$ do not depend on $f$ nor on $\Phi$ and $\Psi$, but only on the weight function $w$.

Inequality (4.5) is an example of a reverse strong-type inequality for the Hardy-Littlewood maximal function. Observe that since $|u| \leq|\mathcal{M} u|$ a.e., it is always true with $\Phi=\Psi$ and an arbitrary weight function $w$. Other results in this direction, for logarithmic spaces $L(\log L)^{k}$, were previously obtained by Favo, Gatto and Gutiérrez [15] and by Anderson and Young [1].

It remains an open problem to describe the class of weights that appear in (4.4) with $\Phi$ and $\Psi$ as in (4.3).

4.2. Interpolation inequalities for derivatives. Using Theorems 4.1 and 4.2 we can now obtain the Gagliardo-Nirenberg inequalities for derivatives in Orlicz spaces.

Our first theorem fits the Bloom-Kerman setting.

Theorem 4.3. Suppose that $\Phi:[0, \infty) \rightarrow[0, \infty)$ and $F:[0, \infty) \rightarrow$ $[0, \infty)$ are two $N$-functions. Let $\mu(d x)=w(x) d x$, where $w$ is a nonnegative weight on $\mathbb{R}^{n}$. Take $k, m \in \mathbb{Z}_{+}, 0<k<m$, and define

$$
\Phi_{1}(\lambda)=\Phi\left(F\left(\lambda^{1-k / m}\right)\right), \quad \Phi_{2}(\lambda)=\Phi\left(F^{*}\left(\lambda^{k / m}\right)\right) .
$$

If $\Phi_{1}, \Phi_{2}$ are $N$-functions of class $\Delta_{2}^{\mathrm{c}}$ and $w \in W_{\Phi_{1}} \cap W_{\Phi_{2}}$ (see Definition 4.1), then for every $f \in C_{0}^{m}\left(\mathbb{R}^{n}\right)$, and arbitrary positive numbers $s, s_{1}, s_{2}$ such that 
$s=s_{1}^{1-k / m} s_{2}^{k / m}$, one has

$$
\begin{gathered}
\int_{\mathbb{R}^{n}} \Phi\left(s\left|\nabla^{(k)} f\right|\right) d \mu \leq \int_{\mathbb{R}^{n}} \Phi_{1}\left(s_{1} B_{1}|f|\right) d \mu+\int_{\mathbb{R}^{n}} \Phi_{2}\left(s_{2} B_{2}\left|\nabla^{(m)} f\right|\right) d \mu, \\
\left\|\nabla^{(k)} f\right\|_{L^{\Phi}(\mu)} \leq B_{3}\|f\|_{L^{\Phi_{1}(\mu)}}^{1-k / m}\left\|\nabla^{(m)} f\right\|_{L^{\Phi_{2}(\mu)}}^{k / m},
\end{gathered}
$$

where the constants $B_{i}$ are independent of $f$ and $s_{i}$.

Proof. As $\Phi$ is increasing, and for every measurable function $u$ one has $|u|<\mathcal{M} u$ a.e., using (3.7) we obtain

$$
\begin{aligned}
\int_{\mathbb{R}^{n}} \Phi\left(\frac{s}{2 C_{0}}\left|\nabla^{(k)} f\right|\right) d \mu & \leq \int_{\mathbb{R}^{n}} \Phi\left(\frac{s}{2 C_{0}} \mathcal{M}\left(\nabla^{(k)} f\right)\right) d \mu \\
& \leq \int_{\mathbb{R}^{n}} \Phi_{1}\left(s_{1} \mathcal{M} f\right) d \mu+\int_{\mathbb{R}^{n}} \Phi_{2}\left(s_{2} \mathcal{M}\left(\nabla^{(m)} f\right)\right) d \mu
\end{aligned}
$$

where $s_{1}, s_{2}>0$ are arbitrary and $s=s_{1}^{1-k / m} s_{2}^{k / m}$. According to Theorem 4.1 , for all measurable $u$ we have $\int_{\mathbb{R}^{n}} \Phi_{i}(\mathcal{M} u) d \mu \leq \int_{\mathbb{R}^{n}} \Phi_{i}\left(B_{\Phi_{i}}|u|\right) d \mu$ for $i=1,2$, which gives

$$
\int_{\mathbb{R}^{n}} \Phi\left(\frac{s}{2 C_{0}}\left|\nabla^{(k)} f\right|\right) d \mu \leq \int_{\mathbb{R}^{n}} \Phi_{1}\left(s_{1} B_{\Phi_{1}}|f|\right) d \mu+\int_{\mathbb{R}^{n}} \Phi_{2}\left(s_{2} B_{\Phi_{2}}\left|\nabla^{(m)} f\right|\right) d \mu .
$$

Now it suffices to substitute $\widetilde{f}=2 C_{0} f$ in the equation above, which results in (4.7) with $B_{i}=2 C_{0} B_{\Phi_{i}}$ where $B_{\Phi_{i}}$ are the constants from Theorem 4.1 and $C_{0}$ is taken from $(3.4)$.

For the proof of (4.8) first note that the inequality $|u| \leq \mathcal{M} u$ implies $\|u\|_{L^{\Phi}(\mu)} \leq\|\mathcal{M} u\|_{L^{\Phi}(\mu)}$. Next, it can be derived from Theorem 4.1 that for an arbitrary measurable function $u$ one has $\|\mathcal{M} u\|_{L^{\Phi_{i}(\mu)}} \leq \widetilde{B}_{i}\|u\|_{L^{\Phi_{i}(\mu)}}$, with $\widetilde{B}_{i}$ independent of $u$, provided $\Phi_{i} \in \Delta_{2}^{\mathrm{c}}$ and $\mu \in W_{\Phi}$. Now (4.8) follows directly from (3.3).

REMARK 4.2 .

(i) Choosing $s=s_{1}=s_{2}=1$ (resp. $s_{1}=B_{1}^{-1}, s_{2}=B_{2}^{-1}$ ) in (4.7) under the assumptions of Theorem 4.3 we get, for all $f \in C_{0}^{m}\left(\mathbb{R}^{n}\right)$,

$$
\int_{\mathbb{R}^{n}} \Phi\left(\left|\nabla^{(k)} f\right|\right) d \mu \leq \int_{\mathbb{R}^{n}} \Phi_{1}\left(B_{1}|f|\right) d \mu+\int_{\mathbb{R}^{n}} \Phi_{2}\left(B_{2}\left|\nabla^{(m)} f\right|\right) d \mu
$$

and

$$
\int_{\mathbb{R}^{n}} \Phi\left(\widetilde{B}\left|\nabla^{(k)} f\right|\right) d \mu \leq \int_{\mathbb{R}^{n}} \Phi_{1}(|f|) d \mu+\int_{\mathbb{R}^{n}} \Phi_{2}\left(\left|\nabla^{(m)} f\right|\right) d \mu .
$$

The constants $\widetilde{B}, B_{1}, B_{2}$ do not depend on $f$. 
(ii) If additionally at least one of the functions $\Phi, \Phi_{1}$ or $\Phi_{2}$ satisfies the $\Delta_{2}$-condition, then

$$
\int_{\mathbb{R}^{n}} \Phi\left(\left|\nabla^{(k)} f\right|\right) d \mu \leq C\left(\int_{\mathbb{R}^{n}} \Phi_{1}(|f|) d \mu+\int_{\mathbb{R}^{n}} \Phi_{2}\left(\left|\nabla^{(m)} f\right|\right) d \mu\right),
$$

with a constant $C$ independent of $f$. See the proof of Corollary 3.1.

If either $\Phi_{1}^{*}$ or $\Phi_{2}^{*}$ does not satisfy the $\Delta_{2}$-condition, then the BloomKerman theorem is not applicable. Instead, we use Kita's results summarized in Theorem 4.2. This leads to the following theorem.

TheOREM 4.4. Let $k, m \in \mathbb{Z}_{+}$with $0<k<m$ and $\mu(d x)=w(x) d x$, where $w \in A_{1} \cap A_{\infty}^{\prime}$. Suppose that $\Phi_{0}:[0, \infty) \rightarrow[0, \infty)$ is an increasing function of class $C^{1}((0, \infty))$ such that $\Phi_{0}(0)=\left(\Phi_{0}\right)_{+}^{\prime}(0)=0$, and that $F$ : $[0, \infty) \rightarrow[0, \infty)$ is an $N$-function of class $C^{1}$ Set

$$
\Phi_{1}(\lambda)=\Phi_{0}\left(F\left(\lambda^{1-k / m}\right)\right), \quad \Phi_{2}(\lambda)=\Phi_{0}\left(F^{*}\left(\lambda^{k / m}\right)\right) .
$$

Assume further that $\int_{0}^{1} \frac{\Phi_{i}(v)}{v^{2}} d v<\infty$ for $i=0,1,2$, and define

$$
\Psi_{i}(\lambda)=\int_{0}^{1} \frac{\Phi_{i}(\lambda v)}{v^{2}} d v, \quad i=0,1,2 .
$$

Then there exist constants $C, K>0$ such that for every $f \in C_{0}^{m}\left(\mathbb{R}^{n}\right)$ and for any positive numbers $s, s_{1}, s_{2}$ such that $s=s_{1}^{1-k / m} s_{2}^{k / m}$ one has

$$
\int_{\mathbb{R}^{n}} \Psi_{0}\left(C s\left|\nabla^{(k)} f\right|\right) d \mu \leq K\left(\int_{\mathbb{R}^{n}} \Psi_{1}\left(s_{1}|f|\right) d \mu+\int_{\mathbb{R}^{n}} \Psi_{2}\left(s_{2}\left|\nabla^{(m)} f\right|\right) d \mu\right) .
$$

Moreover, if $\Psi_{1}$ and $\Psi_{2}$ are $N$-functions, then also

$$
\left\|\nabla^{(k)} f\right\|_{L^{\Psi_{0}(\mu)}} \leq \widetilde{K}\|f\|_{L^{\Psi_{1}(\mu)}}^{1-k / m}\left\|\nabla^{(m)} f\right\|_{L^{\Psi_{2}(\mu)}}^{k / m}
$$

with the constant $\widetilde{K}$ independent of $f$.

REMARK 4.3. The constant $C$ in (4.13) is taken from (3.2) and depends on $n$ only.

Proof. By (3.7), we have

$$
\begin{aligned}
I & :=\int_{\mathbb{R}^{n}} \Phi_{0}\left(\frac{s}{2 C_{0}} \mathcal{M}\left(\nabla^{(k)} f\right)\right) d \mu \\
& \leq \int_{\mathbb{R}^{n}} \Phi_{1}\left(s_{1} \mathcal{M} f\right) d \mu+\int_{\mathbb{R}^{n}} \Phi_{2}\left(s_{2} \mathcal{M}\left(\nabla^{(m)} f\right)\right) d \mu=: I_{1}+I_{2},
\end{aligned}
$$

where $s_{1}, s_{2}>0$ are arbitrary and $s=s_{1}^{1-k / m} s_{2}^{k / m}$. An easy computation shows that $\Psi_{i}^{\prime}(\lambda)=\int_{0}^{\lambda}\left(\Phi_{i}^{\prime}(\tau) / \tau\right) d \tau$, and $\Psi_{i}^{\prime \prime}(\lambda)=\Phi_{i}^{\prime}(\lambda) / \lambda$ for $i=0,1,2$. 
Therefore the assumptions of Theorem 4.2 are satisfied for pairs $\left(\Phi_{i}, \Psi_{i}\right)$ with $i=0,1,2$. This implies

$$
\begin{gathered}
\int_{\mathbb{R}^{n}} \Psi_{0}\left(\frac{s}{2 C_{0}}\left|\nabla^{(k)} f\right|\right) d \mu \leq K_{2} I, \\
I_{1} \leq K_{1} \int_{\mathbb{R}^{n}} \Psi_{1}\left(s_{1}|f|\right) d \mu, \quad I_{2} \leq K_{1} \int_{\mathbb{R}^{n}} \Psi_{2}\left(s_{2}\left|\nabla^{(m)} f\right|\right) d \mu,
\end{gathered}
$$

and (4.13) follows with $K=K_{1} K_{2}$, where $K_{1}$ and $K_{2}$ are the constants from (4.4) and (4.5). To get (4.14), we assume that both summands on the right-hand side of (4.13) are nonzero; then we use (4.13), choosing $s_{1}=$ $\left(\|f\|_{L^{\Psi_{1}(\mu)}}\right)^{-1}$ and $s_{2}=\left(\left\|\nabla^{(m)} f\right\|_{L^{\Psi_{2}(\mu)}}\right)^{-1}$. This results in

$$
\frac{1}{2 K} \int_{\mathbb{R}^{n}} \Phi_{0}\left(\frac{s\left|\nabla^{(k)} f\right|}{2 C_{0}}\right) d \mu \leq 1 .
$$

Clearly $\frac{1}{2 K} \Psi_{0}\left(\frac{\lambda}{2 C_{0}}\right) \asymp \Psi_{0}(\lambda)$, and so (4.14) follows directly from (2.6), with $\widetilde{K}=2 C_{0}(2 K+1)$.

REMARK 4.4 .

(i) Taking $s_{1}=s_{2}=1$ in (4.13) we get

$$
\int_{\mathbb{R}^{n}} \Psi_{0}\left(C\left|\nabla^{(k)} f\right|\right) d \mu \leq K\left(\int_{\mathbb{R}^{n}} \Psi_{1}(|f|) d \mu+\int_{\mathbb{R}^{n}} \Psi_{2}\left(\left|\nabla^{(m)} f\right|\right) d \mu\right),
$$

where $C$ and $K$ are independent of $f$.

(ii) If the assumptions of Theorem 4.4 are satisfied, and additionally at least one of the functions $\Psi_{0}, \Psi_{1}$ or $\Psi_{2}$ satisfies the $\Delta_{2}$-condition, then

$$
\int_{\mathbb{R}^{n}} \Psi_{0}\left(\left|\nabla^{(k)} f\right|\right) d \mu \leq C\left(\int_{\mathbb{R}^{n}} \Psi_{1}(|f|) d \mu+\int_{\mathbb{R}^{n}} \Psi_{2}\left(\left|\nabla^{(m)} f\right|\right) d \mu\right),
$$

with a constant $C$ independent of $f$. Arguments are similar to those in Corollary 3.1 and Remark 4.2.

Examples of triples $\left(\Psi_{0}, \Psi_{1}, \Psi_{2}\right)$ satisfying (4.13) and (4.14) are given in Section 6.

\section{Further discussion. Comments and remarks}

5.1. Additional comments concerning Theorems 4.3 and 4.4. We start with the following auxiliary observations concerning functions that appear in Kita's theorem (Theorem 4.2).

Proposition 5.1. Suppose that $\Phi$ and $\Psi$ are defined by (4.3), with a and $b$ satisfying the assumptions of Theorem 4.2. Then we have:

(1) $\Phi$ is nondecreasing, $\Psi$ is convex, and $\Phi(0)=\Psi(0)=0$. For every $t>0$ we have $\Phi \in W^{1,1}(0, t)$ and $\Psi \in W^{2,1}(0, t)$. 
(2) $\Phi$ and $\Psi$ can be related through

$$
\Phi(t)=t \Psi^{\prime}(t)-\Psi(t) .
$$

If $\Psi$ is strictly convex, then $\Phi(t)=\Psi^{*}\left(\Psi^{\prime}(t)\right)$.

(3) $\Phi$ and $\Psi$ can also be related through an integral identity

$$
\Psi(\lambda)=\lambda \int_{0}^{\lambda} \frac{\Phi(v)}{v^{2}} d v=\int_{0}^{1} \frac{\Phi(\lambda v)}{v^{2}} d v
$$

$$
\Phi(t) \leq \Psi(2 t) \text { for all } t>0 .
$$

(5) If $a(t)>0$ for $t>0$, then $\Psi^{*}$ satisfies the $\Delta_{2}$-condition if and only if $\Phi(t) \geq \beta \Psi(t)$ for some $\beta>0$.

(6) If $a(t)>0$ for $t>0$, then $\Psi \in \Delta_{2}^{c}$ if and only if $\Phi$ and $\Psi$ are equivalent $N$-functions.

(7) If $a(t)>0$ for $t>0$, then $\Phi \in \Delta_{2}^{\mathrm{c}}$ if and only if $\Psi \in \Delta_{2}^{\mathrm{c}}$.

Proof. (1) follows directly from the definition and (2) is verified by elementary differentiation.

Let us prove (3). Reversing the order of integration in the definition of $\Psi$, we have

$$
\Psi(\lambda)=\int_{0}^{\lambda} \int_{0}^{t} \frac{\Phi^{\prime}(u)}{u} d u d t=\lambda \int_{0}^{\lambda} \frac{\Phi^{\prime}(u)}{u} d u-\Phi(\lambda) .
$$

As

$$
\frac{\Phi(\lambda)}{\lambda}=\frac{1}{\lambda} \int_{0}^{\lambda} a(\tau) d \tau \leq \int_{0}^{\lambda} \frac{a(\tau)}{\tau} d \tau,
$$

we see that $\lim _{\lambda \rightarrow 0} \Phi(\lambda) / \lambda=0$. Integrating (5.4) by parts we obtain $\Psi(\lambda)=$ $\lambda \int_{0}^{\lambda}\left(\Phi(u) / u^{2}\right) d u$. A simple substitution $u=\lambda v$ in this integral gives (5.2).

To see (4), observe that

$$
\Phi(t)=t \Psi^{\prime}(t)-\Psi(t) \leq \int_{t}^{2 t} \Psi^{\prime}(u) d u-\Psi(t)=\Psi(2 t)-2 \Psi(t),
$$

i.e. $\Phi(t)+2 \Psi(t) \leq \Psi(2 t)$.

(5) follows from the identity

$$
\frac{t \Psi^{\prime}(t)}{\Psi(t)}=\frac{\Phi(t)+\Psi(t)}{\Psi(t)}=1+\frac{\Phi(t)}{\Psi(t)}
$$

and Proposition 4.1.

The "if" part of (6) is obtained directly from (4) and (5). For the converse, assume that $\Phi \asymp \Psi$. Since the Lebesgue measure belongs to $A_{1}$, from Kita's 
theorem we get

$$
\int \Phi(\mathcal{M} f(x)) d x \leq K_{1} \int \Psi(|f(x)|) d x
$$

which yields

$$
\int \Phi(\mathcal{M} f(x)) d x \leq \widetilde{K}_{1} \int \Phi(C|f(x)|) d x .
$$

Next, as for every convex function such that $\Phi(0)=0$ and for every $N>1$ one has $\Phi(N \lambda) \geq N \Phi(\lambda)$, the constant $\widetilde{K}_{1}$ can be incorporated into the constant $C$ inside the argument of $\Phi$, and we obtain exactly (4.1). Therefore from the Bloom-Kerman theorem (Theorem 4.1) we can infer that $\Phi \in \Delta_{2}^{\mathrm{c}}$. As $\Phi \asymp \Psi$, we have $\Psi \in \Delta_{2}^{\mathrm{c}}$, which follows from (D3) of Proposition 4.1. So (6) is proven.

To see (7), we only need to show that $\Phi \in \Delta_{2}^{\mathrm{c}}$ implies $\Phi \asymp \Psi$. But this is clear: the inequality $\Phi(t) \leq \Psi(2 t)$ is always true, and the reverse domination follows from Proposition 4.1. Indeed, $\Phi \in \Delta_{2}^{c}$ is equivalent to the fact that $\Phi(\lambda) / \lambda^{\alpha}$ is nondecreasing for some $\alpha>1$. Therefore

$$
\Psi(\lambda)=\lambda \int_{0}^{\lambda} \frac{\Psi(t)}{t^{\alpha}} \frac{1}{t^{2-\alpha}} d t \leq \lambda^{1-\alpha} \Phi(\lambda) \int_{0}^{\lambda} \frac{1}{t^{2-\alpha}} d t=\frac{1}{\alpha-1} \Phi(\lambda) .
$$

Now we make the following remarks.

REMARK 5.1. The representation (5.2) was used in formula (4.12).

REMARK 5.2. Let $d \mu(x)=w(x) d x$ be a weighted measure and let $\Psi \in \Delta_{2}^{\mathrm{c}}$ be an increasing function. Then from Proposition 5.1(6), we can infer that $\Phi$ and $\Psi$ are equivalent. According to Kita's results (Theorem 4.2) we see that the Hardy-Littlewood maximal operator satisfies an inequality of the form

$$
\begin{aligned}
\int_{\mathbb{R}^{n}} \Psi(\mathcal{M} f(x)) w(x) d x & \leq C_{1} \int_{\mathbb{R}^{n}} \Psi\left(C_{2}|f(x)|\right) w(x) d x \\
& \leq \int_{\mathbb{R}^{n}} \Psi\left(C_{3}|f(x)|\right) w(x) d x
\end{aligned}
$$

with constants $C_{1}, C_{2}, C_{3}$ independent of $f$, provided $w \in A_{1} \cap A_{\infty}^{\prime}$. Theorem 4.1 gives more, namely that (5.5) is satisfied whenever $w \in W_{\Psi}$.

Note that, as $A_{1}=\bigcap_{\Psi} W_{\Psi}$, the class $W_{\Psi}$ is substantially larger than $A_{1} \cap A_{\infty}^{\prime}$. Therefore, when $\Psi \in \Delta_{2}^{\mathrm{c}}$, Theorem 4.1 extends Theorem 4.2 to a wider class of measures.

REMARK 5.3. For measures $\mu \in A_{1} \cap A_{\infty}^{\prime}$, Theorem 4.4 implies the results of Theorem 4.3 .

Indeed, suppose that $\left(\Phi_{0}, \Phi_{1}, \Phi_{2}\right)$ and $\left(\Psi_{0}, \Psi_{1}, \Psi_{2}\right)$ are two triples of functions that appear in Theorem 4.4. Assume $\mu(d x)=w(x) d x, w \in A_{1} \cap A_{\infty}^{\prime}$, 
$1<k<m, s_{1}, s_{1}>0, s=s_{1}^{1-k / m} s_{2}^{k / m}$. Then the inequalities

$$
\int_{\mathbb{R}^{n}} \Psi_{0}\left(C s\left|\nabla^{(k)} f\right|\right) d \mu \leq K\left(\int_{\mathbb{R}^{n}} \Psi_{1}\left(s_{1}|f|\right) d \mu+\int_{\mathbb{R}^{n}} \Psi_{2}\left(s_{2}\left|\nabla^{(m)} f\right|\right) d \mu\right)
$$

and

$$
\left\|\nabla^{(k)} f\right\|_{L^{\Psi_{0}(\mu)}} \leq \widetilde{K}\|f\|_{L^{\Psi_{1}(\mu)}}^{1-k / m}\left\|\nabla^{(m)} f\right\|_{L^{\Psi_{2}(\mu)}}^{k / m},
$$

hold with constants $C, K, \widetilde{K}$ independent of $f$. By Proposition 5.1(4), for every $\lambda>0$ one has $\Phi_{0}(\lambda) \leq \Psi_{0}(2 \lambda)$, and so

$$
\int \Phi_{0}\left(\frac{C s}{2}\left|\nabla^{(k)} f\right|\right) d \mu \leq \int \Psi_{0}\left(C s\left|\nabla^{(k)} f\right|\right) d \mu .
$$

Suppose additionally that $\Phi_{1}, \Phi_{2} \in \Delta_{2}^{\mathrm{c}}$, so that the assumptions of Theorem 4.4 are satisfied. By Proposition 5.1(6) we have $\Psi_{i} \asymp \Phi_{i}$, thus $\Psi_{i} \in \Delta_{2}^{\mathrm{c}}$ as well. Therefore, by Proposition 5.1(5), we get $\beta_{i} \Psi_{i}(\lambda) \leq \Phi_{i}(\lambda)$. These observations (used for $(2 / C) f$ instead of $f$ ) lead to the inequalities

$$
\int \Phi_{0}\left(s\left|\nabla^{(k)} f\right|\right) d \mu \leq K_{1}\left(\int \Phi_{1}\left(s_{1} \widetilde{B}_{1}|f|\right) d \mu+\int \Phi_{2}\left(s_{2} \widetilde{B}_{2}\left|\nabla^{(m)} f\right|\right) d \mu\right)
$$

and

$$
\left\|\nabla^{(k)} f\right\|_{L^{\Phi_{0}(\mu)}} \leq \widetilde{K}_{1}\|f\|_{L^{\Phi_{1}(\mu)}}^{1-k / m}\left\|\nabla^{(m)} f\right\|_{L^{\Phi_{2}(\mu)}}^{k / m},
$$

which are the same as (4.7) and (4.8), up to multiplicative constants.

REMARK 5.4. For some functions $\Phi$ for which the assumptions of Theorem 4.3 fail, Theorem 4.4 can still be applied. For example, suppose that the function $\Phi$ in Theorem 4.3 does not satisfy the $\Delta_{2}^{\mathrm{c}}$-condition. Choose $F(\lambda)=\lambda^{m /(m-k)}$. Then $\Phi_{1}(\lambda)=\Phi(\lambda)$ and $\Phi_{2}(\lambda)=\Phi(c \lambda)$ do not satisfy the $\Delta_{2}^{\mathrm{c}}$-condition either. Therefore Theorem 4.3 cannot be applied to obtain Gagliardo-Nirenberg inequalities within the single Orlicz space $L^{\Phi}(\mu)$ (the Landau-Kolmogorov inequalities). But in this case Theorem 4.4 can be used and yields the desired inequality, at least for measures $\mu \in A_{1} \cap A_{\infty}^{\prime}$.

6. Examples. We start with a single Orlicz space $L^{\Psi}(\mu)$.

EXAMPLE 6.1 (Kolmogorov-Stein inequalities). Suppose that $\mu(d x)=$ $w(x) d x$ and either $\left\{\Psi \in \Delta_{2}^{\mathrm{c}}\right.$ and $\left.w \in W_{\Psi}\right\}$ or $\{\Psi$ is an arbitrary N-function and $\left.w \in A_{1} \cap A_{\infty}^{\prime}\right\}$. Take $F(\lambda)=\lambda^{m /(m-k)}$, so that $F^{*}(\lambda)=c \lambda^{m / k}$ with $c=c_{k, m}<1$. Then we get $\Psi_{1}(\lambda)=\Psi(\lambda)$ and $\Psi_{2}(\lambda)=\Psi(c \lambda)$ with a constant $c<1$. Therefore it follows from Theorems 4.3 and 4.4 that in either case we have

$$
\int_{\mathbb{R}^{n}} \Psi\left(C s\left|\nabla^{(k)} f\right|\right) d \mu \leq K\left(\int_{\mathbb{R}^{n}} \Psi\left(s_{1}|f|\right) d \mu+\int_{\mathbb{R}^{n}} \Psi\left(s_{2}\left|\nabla^{(m)} f\right|\right) d \mu\right)
$$


for every $f \in C_{0}^{m}\left(\mathbb{R}^{n}\right)$ and arbitrary $s, s_{1}, s_{2}>0$ such that $s=s_{1}^{1-k / m} s_{2}^{k / m}$, with constants $C, K$ independent of $f$. Furthermore,

$$
\left\|\nabla^{(k)} f\right\|_{L^{\Psi}(\mu)} \leq \widetilde{C}\|f\|_{L^{\Psi}(\mu)}^{1-k / m}\left\|\nabla^{(m)} f\right\|_{L^{\Psi}(\mu)}^{k / m}
$$

with a constant $\widetilde{C}$ independent of $f$. Inequalities (6.2) for a one-variable function $f$ and the Lebesgue measure were recently obtained by Bang and coauthors [3] directly from the classical Kolmogorov inequality, by means of the convolution technique due to Stein.

EXAmple 6.2 (Gagliardo-Nirenberg inequalities). Take $\Phi(\lambda)=\lambda^{q}$, let $p, r>1$ be such that

$$
\frac{1}{q}=\left(1-\frac{k}{m}\right) \frac{1}{p}+\frac{k}{m} \frac{1}{r}
$$

and let

$$
F(\lambda)=\frac{1}{s} \lambda^{s} \quad \text { with } \quad s=\frac{m}{m-k} \frac{p}{q}
$$

Then

$$
F^{*}(\lambda)=\frac{1}{s^{*}} \lambda^{s^{*}} \quad \text { and } \quad s^{*}=\frac{m}{k} \frac{r}{q} .
$$

According to Theorem 4.3 we have $\Phi_{1}(\lambda) \asymp \lambda^{p}, \Phi_{2}(\lambda) \asymp \lambda^{r}$ and all these functions, together with their Legendre transforms, satisfy the $\Delta_{2}$-condition. Recalling that for $\Phi(\lambda)=|\lambda|^{\kappa}$ we have $W_{\Phi}=A_{\kappa}$ where $A_{\kappa}$ is the Muckenhoupt class (see e.g. [44]), by Theorem 4.3 and Remark 4.2 we obtain

$$
\int_{\mathbb{R}^{n}}|\nabla f|^{q} d \mu \leq C\left(\int_{\mathbb{R}^{n}}|f|^{p} d \mu+\int_{\mathbb{R}^{n}}\left|\nabla^{(2)} f\right|^{r} d \mu\right)
$$

and also

$$
\left\|\nabla^{(k)} f\right\|_{L^{q}(\mu)} \leq C\|f\|_{L^{p}(\mu)}^{1-k / m}\left\|\nabla^{(m)} f\right\|_{L^{r}(\mu)}^{k / m},
$$

provided that $\mu \in A_{p} \cap A_{r}$, with a constant $C$ independent of $f$. These and other general interpolation inequalities in weighted $L^{\kappa}$ spaces equipped with Muckenhoupt weights were previously obtained by the first author in [25].

Our next example deals with the case when $\Phi \in \Delta_{2} \cap \Delta_{2}^{\mathrm{c}}$ and it is not homogeneous.

EXAmple 6.3 (logarithmic inequalities). For $s>1$ and $\kappa \in \mathbb{R}$ let

$$
M_{s, \kappa}(\lambda)=\lambda^{s} \ln (2+\lambda)^{\kappa} .
$$

Suppose that $\mu(d x)=w(x) d x$ is a nonnegative weighted measure on $\mathbb{R}^{n}$. Write $\|\cdot\|_{(s, \kappa, \mu)}=\|\cdot\|_{\left(M_{(s, \kappa)}, \mu\right)}$, where $\mu$ is dropped from the notation if it is the Lebesgue measure. In this case we obtain the following result. 
Theorem 6.1. Let $k, m \in \mathbb{Z}_{+}$with $0<k<m$. Suppose that $p, q, r>1$ and $\alpha, \beta, \gamma \in \mathbb{R}$ satisfy the conditions

$$
\frac{1}{q}=\left(1-\frac{k}{m}\right) \frac{1}{p}+\frac{k}{m} \frac{1}{r}, \quad \frac{\alpha}{q}=\left(1-\frac{k}{m}\right) \frac{\beta}{p}+\frac{k}{m} \frac{\gamma}{r},
$$

and let $\mu(d x)=w(x) d x$ be a weighted measure with $w \in W_{M_{p, \beta}} \cap W_{M_{r, \gamma}}$. Then for every function $f \in C_{0}^{m}\left(\mathbb{R}^{n}\right)$ we have

$$
\begin{aligned}
& \int\left|\nabla^{(k)} f\right|^{q} \ln \left(2+\left|\nabla^{(k)} f\right|\right)^{\alpha} d \mu \\
& \quad \leq C\left(\int|f|^{p} \ln (2+|f|)^{\beta} d \mu+\int\left|\nabla^{(m)} f\right|^{r} \ln \left(2+\left|\nabla^{(m)} f\right|\right)^{\gamma} d \mu\right),
\end{aligned}
$$

and also

$$
\left\|\nabla^{(k)} f\right\|_{(q, \alpha, \mu)} \leq C\|f\|_{(p, \beta, \mu)}^{1-k / m}\left\|\nabla^{(m)} f\right\|_{(r, \gamma, \mu)}^{k / m},
$$

with a constant $C$ independent of $f$.

Proof. It is clear that

$$
M_{s_{1}, \kappa_{1}} \circ M_{s_{2}, \kappa_{2}}(\lambda) \asymp \lambda^{s_{1} s_{2}}(\ln (2+\lambda))^{\kappa_{1}+s_{1} \kappa_{2}}=M_{s_{1} s_{2}, \kappa_{1}+s_{2} \kappa_{2}}(\lambda) .
$$

Also, it is elementary to check that $M_{s, \kappa}^{*} \asymp M_{s^{*},-\kappa\left(s^{*}-2\right)}$. This is so because $M_{s, \kappa}(\lambda) \asymp \lambda^{s}$ for $\lambda$ small, and $M_{s, \kappa}(\lambda) \asymp \lambda^{s}(\ln \lambda)^{\kappa}$ for large values of $\lambda$. Consequently, $M_{s, \kappa}^{*}(\lambda) \asymp \lambda^{s^{*}}$ for $\lambda$ small and $M_{s, \kappa}^{*}(\lambda) \asymp \lambda^{s^{*}}(\ln \lambda)^{-\kappa\left(s^{*}-1\right)}$ for $\lambda$ large (see Theorem 7.1 of [35]).

Suppose now that $q, p, r, \alpha, \beta, \gamma$ satisfy (6.3). Then one applies Theorem 4.3 for $\Phi=M_{q, \alpha}$ and $F=M_{s, \kappa}$, which is allowed as all the functions considered belong to the class $\Delta_{2}^{\mathrm{c}}$. From the just proven properties of the functions $M_{s, \kappa}$ one verifies that

$\Phi_{1}(\lambda) \asymp \lambda^{s q(1-k / m)}(\ln (2+\lambda))^{\alpha+\kappa q}, \quad \Phi_{2}(\lambda) \asymp \lambda^{s^{*} q k / m}(\ln (2+\lambda))^{\alpha-q \kappa\left(s^{*}-1\right)}$, and choosing $s=\frac{p}{q} \frac{m}{m-k}, \kappa=\frac{\beta-\alpha}{q}$ one gets $\Phi_{1}(\lambda) \asymp M_{p, \beta}(\lambda)$ and $\Phi_{2}(\lambda) \asymp$ $M_{r, \gamma}(\lambda)$. The theorem is proven.

When $w(x) \equiv 1$ this result, but within a narrower class of parameters, was obtained in [28], as a special case of more general inequalities of the form (1.3), (1.4) adapted to logarithmic spaces.

We also refer to that paper for some details about logarithmic-type Nfunctions that were only sketched above. Finally, note that for $\alpha=\mu=\gamma$ $=0$ we get the Gagliardo-Nirenberg inequalities discussed in Example 6.2.

In our next example all the functions $\Phi, \Phi_{1}$ and $\Phi_{2}$ belong to the class $\Delta_{2}^{\mathrm{c}}$, but none of them satisfies the $\Delta_{2}$-condition.

ExAmple 6.4 (exponential inequalities). Now we present inequalities for exponential N-functions. 
Theorem 6.2. Let $k, m \in \mathbb{Z}_{+}$with $0<k<m$. Suppose that $p, q, r>1$ and $\alpha, \beta, \gamma>0$ satisfy the following conditions:

$$
\frac{1}{q}=\left(1-\frac{k}{m}\right) \frac{1}{p}+\frac{k}{m} \frac{1}{r}, \quad \frac{1}{\alpha}=\left(1-\frac{k}{m}\right) \frac{1}{\beta}+\frac{k}{m} \frac{1}{\gamma} .
$$

Then for every $f \in C_{0}^{m}\left(\mathbb{R}^{n}\right)$ one has

$$
\begin{aligned}
& \int_{\mathbb{R}^{n}}\left|\nabla^{(k)} f\right|^{q} \exp \left(\left|\nabla^{(k)} f\right|^{\alpha}\right) d \mu \\
& \quad \leq C\left(\int_{\mathbb{R}^{n}}|f|^{p} \exp \left(C_{1}|f|^{\beta}\right) d \mu+\int_{\mathbb{R}^{n}}\left|\nabla^{(m)} f\right|^{r} \exp \left(C_{2}\left|\nabla^{(m)} f\right|^{\gamma}\right) d \mu\right) ;
\end{aligned}
$$

and also

$$
\left\|\nabla^{(k)} f\right\|_{L^{\Phi}(\mu)} \leq \widetilde{C}_{3}\|f\|_{L^{\Phi_{1}(\mu)}}^{1-k / m}\left\|\nabla^{(m)} f\right\|_{L^{\Phi_{2}(\mu)}}^{k / m},
$$

where the constants $C, C_{1}, C_{2}, C_{3}$ are independent of $f$,

$$
\Phi(\lambda)=\lambda^{q} \exp \left(\lambda^{\alpha}\right), \quad \Phi_{1}(\lambda)=\lambda^{p} \exp \left(\lambda^{\beta}\right), \quad \Phi_{2}(\lambda)=\lambda^{r} \exp \left(\lambda^{\gamma}\right),
$$

and $\mu(x)=w(x) d x$, where $w \in W_{\Phi_{1}} \cap W_{\Phi_{2}}$.

Proof. Take $F(\lambda)=\lambda^{s_{1}} \exp \left(\left(s_{2}-s_{1}\right) \ln (2+\lambda)\right)$, where the parameters $s_{1}, s_{2}>1$ will be determined later. Then $F$ is an N-function, and moreover, $F(\lambda) \sim \lambda^{s_{1}}$ for $\lambda$ close to 0 and $F(\lambda) \sim \lambda^{s_{2}}$ for $\lambda$ close to $\infty$. Hence $F^{*}(\lambda) \sim \lambda^{s_{1}^{*}}$ for $\lambda$ close to 0 and $F^{*}(\lambda) \sim \lambda^{s_{2}^{*}}$ for $\lambda$ close to $\infty$. On the other hand, we have $\Phi(\lambda) \sim \lambda^{q}$ for $\lambda$ close to 0 and $\Phi(\lambda) \sim \exp \left(\lambda^{\alpha}\right)$ for $\lambda$ close to $\infty$. Therefore if we take $s_{1}=p / q(1-k / m), s_{2}=\beta /(1-k / m) \alpha$ and use condition (6.5) we obtain

$$
\Phi_{1}(\lambda) \sim \bar{\Phi}_{1}(\lambda)=: \Phi\left(F\left(\lambda^{1-k / m}\right)\right), \quad \Phi_{2}(\lambda) \sim \bar{\Phi}_{2}(\lambda)=: \Phi\left(F^{*}\left(\lambda^{k / m}\right)\right) .
$$

Next, as $w \in W_{\Phi_{1}} \cap W_{\Phi_{2}}$, according to Remark 4.1, we see that $\omega \in$ $W_{\bar{\Phi}_{1}} \cap W_{\bar{\Phi}_{2}}$. Moreover, by Proposition 4.1 we have $\Phi_{1}, \Phi_{2} \in \Delta_{2}^{\mathrm{c}}$ and therefore $\bar{\Phi}_{1}, \bar{\Phi}_{2} \in \Delta_{2}^{\mathrm{c}}$ as well. Hence, it suffices to apply Theorem 4.3 with the functions $\left(\Phi, \bar{\Phi}_{1}, \bar{\Phi}_{2}\right)$ and again use the fact that $\Phi_{1} \sim \bar{\Phi}_{2}$ and $\Phi_{2} \sim \bar{\Phi}_{2}$.

REMARK 6.1. In our previous paper (see Example 8.7 in [27]), from more general inequalities we derived inequalities similar to (6.6) and (6.7) for $k=1, m=2$, but with parameters $p, q, r, \alpha, \beta, \gamma$ satisfying

$$
q>2, \alpha \in(0,2), \frac{1}{2 p}+\frac{1}{2 r}=\frac{1}{q}, \beta=\frac{s \alpha}{2-\alpha}, \gamma=\frac{s^{*} \alpha}{2-\alpha} \text { and } s=\frac{2 p}{q} .
$$

In particular, for those parameters we have

$$
\frac{1}{2 \beta}+\frac{1}{2 \gamma}=\frac{2-\alpha}{2 \alpha}<\frac{1}{\alpha}
$$

This shows that inequalities (6.6) and (6.7) obtained in Theorem 6.2 (reduced to $k=1, m=2, \mu \equiv d x, p>2, \alpha \in(0,2))$ are valid for a broader range of parameters than the exponential inequalities from [27]. 


\section{Appendix}

7.1. Proof of Proposition 4.1. It is a slight modification of the proof of [35, Theorem 4.3], where the $\Delta_{2}$-condition was assumed only for large values of $t$.

As $\left(\frac{\Phi(t)}{t^{\alpha}}\right)^{\prime}=\frac{t \Phi^{\prime}(t)-\alpha \Phi(t)}{t^{\alpha+1}}$, the equivalence (D2) $\Leftrightarrow(\mathrm{D} 3)$ is obvious.

Set $\Phi^{\prime}(t)=p(t),\left(\Phi^{*}\right)^{\prime}(s)=q(s)=p^{-1}(s)$. Then

$$
\Phi(t)=t p(t)-\Phi^{*}(p(t)) \quad \text { for } t>0,
$$

and similarly

$$
\Phi^{*}(s)=s q(s)-\Phi(q(s)) \quad \text { for } s>0 .
$$

To see that $(\mathrm{D} 1) \Rightarrow(\mathrm{D} 2)$, suppose that $\Phi^{*}$ satisfies the $\Delta_{2}$-condition, i.e. there exists $\beta>0$ such that

$$
\frac{s q(s)}{\Phi^{*}(s)} \leq \beta \quad \text { for every } s>0 .
$$

As $\Phi^{*}$ is strictly convex, we have $s q(s)>\Phi^{*}(s)$, so that $\beta>1$. Inserting (7.1) into (7.2) gives

$$
\frac{s q(s)}{s q(s)-\Phi(q(s))} \leq \beta .
$$

As for $t=q(s)$ one has $s q(s)=t p(t)$, we get

and further

$$
\frac{t p(t)}{t p(t)-\Phi(t)} \leq \beta
$$

$$
\frac{t \Phi^{\prime}(t)}{\Phi(t)} \geq \frac{\beta}{\beta-1}=: \alpha>1 .
$$

It is clear that this reasoning can be reversed, proving also the implication (D2) $\Rightarrow(\mathrm{D} 1)$.

7.2. Proof of Theorem 4.2. We start with two lemmas. For the proof of the first one we refer for example to [31, Lemma 3.2].

Lemma 7.1. Suppose $w \in A_{1}$ and $\mu=\omega d x$. Then for each $f \in \mathcal{R}_{0}(\mu)$ (see (4.2)) one has

$$
\mu\{\mathcal{M} f>t\} \leq \frac{C}{t} \int_{t / 2}^{\infty} \mu\{|f|>s\} d s \quad \text { for all } t>0
$$

where $C$ is a constant independent of $f$.

The other lemma is due to Muckenhoupt [44].

Lemma 7.2. Suppose $w \in A_{\infty}^{\prime}$. Then for each $f \in \mathcal{R}_{0}(\mu)$ one has

$$
\mu\{\mathcal{M} f>t\} \geq \frac{C}{t} \int_{t}^{\infty} \mu\{|f|>s\} d s \quad \text { for all } t>0,
$$

where $C$ is a constant independent of $f$. 
Proof of Theorem 4.2. For a measurable $f$ we can write

$$
\int_{\mathbb{R}^{n}} \Phi(\mathcal{M} f) d \mu=\int_{(0, \infty)} \Phi^{\prime}(s) \mu\{\mathcal{M} f>s\} d s
$$

(these integrals are either simultaneously infinite, or both finite and equal). By Lemma 7.1 this is further equal to

$$
\begin{aligned}
\int_{(0, \infty)} a(s) \mu\{\mathcal{M} f>s\} d s & \leq C \int_{0}^{\infty} \frac{a(s)}{s}\left(\int_{s}^{\infty} \mu\{|f|>t\} d t\right) d s \\
& =C \int_{0}^{\infty} \mu\{|f|>t\}\left(\int_{0}^{t} \frac{a(s)}{s} d s\right) d t \\
& =C \int_{0}^{\infty} b(t) \mu\{|f|>t\} d t \\
& =C \int_{0}^{\infty} \Psi^{\prime}(t) \mu\{|f|>t\} d t=C \int_{\mathbb{R}^{n}} \Psi(|f|) d \mu
\end{aligned}
$$

and (4.4) is proven.

It is clear that for $w \in A_{\infty}^{\prime}$ this chain of inequalities can be reversed (use Lemma 7.2 instead of Lemma 7.1), giving (4.5).

REMARK 7.1. Note that the constants $K_{1}$ and $K_{2}$ do not depend on $\Phi$ and $\Psi$.

\section{References}

[1] K. F. Anderson and W. Young, On the reverse weak type inequality for the Hardy maximal function and the weighted class $L(\log L)^{k}$, Pacific J. Math. 112 (1984), $257-264$.

[2] R. J. Bagby and J. D. Parsons, Orlicz spaces and rearranged maximal functions, Math. Nachr. 132 (1987), 15-27.

[3] H. H. Bang, A remark on the Kolmogorov-Stein inequality, J. Math. Anal. Appl. 203 (1996), 861-867.

[4] H. H. Bang and H. M. Giao, On the Kolmogorov inequality for $M_{\Phi}$-norm, Appl. Anal. 81 (2002), 1-11.

[5] H. H. Bang and H. M. Le, On an inequality of Kolmogorov and Stein, Bull. Austral. Math. Soc. 61 (2000), 153-159.

[6] H. H. Bang and M. T. Thu, A Landau-Kolmogorov inequality for Orlicz spaces, J. Inequal. Appl. 7 (2002), 663-672.

[7] O. V. Besov, V. P. Il'in and S. M. Nikol'skiř, Integral Representations of Functions and Embedding Theorems, Nauka, Moscow, 1975 (in Russian).

[8] S. Bloom and R. Kerman, Weighted Orlicz space integral inequalities for the HardyLittlewood maximal operator, Studia Math. 110 (1994), 149-167.

[9] J. Boman, Supremum norm estimates for partial derivatives of functions of several real variables, Illinois J. Math. 16 (1972), 203-216. 
[10] K. Chełmiński, On monotone plastic constitutive equations with polynomial growth condition, Math. Meth. Appl. Sci. 22 (1999), 547-562.

[11] S. K. Chua, On weighted Sobolev interpolation inequalities, Proc. Amer. Math. Soc. 121 (1994), 441-449.

[12] - Weighted Sobolev interpolation inequalities on certain domains, J. London Math. Soc. 51 (1995), 532-544.

[13] A. Cianchi, Strong and weak type inequalities for some classical operators in Orlicz spaces, ibid. 60 (1999), 203-223.

[14] - Some results in the theory of Orlicz spaces and applications to variational problems, in: Nonlinear Analysis, Function Spaces and Applications, Vol. 6 (Prague, 1998), Acad. Sci. Czech Rep., Prague, 1999, 50-92.

[15] N. A. Favo, E. A. Gatto and C. Gutiérrez, On the strong maximal function and Zygmund's class $L\left(\log ^{+} L\right)^{n}$, Studia Math. 69 (1980), 155-158.

[16] E. Gagliardo, Ulteriori proprietà di alcune classi di funzioni in più variabili, Ricerche Mat. 8 (1959), 24-51.

[17] L. Greco, T. Iwaniec and B. Moscariello, Limits of the improved inequality of the volume forms, Indiana Univ. Math. J. 44 (1995), 305-339.

[18] C. E. Gutiérrez and R. L. Wheeden, Sobolev interpolation inequalities with weights, Trans. Amer. Math. Soc. 323 (1991), 263-281.

[19] J. Hadamard, Sur certaines propriétés des trajectoires en dynamique, Mémoire couronné en 1896 par l'Académie: Prix Bordin, J. Math. (5) 3, 331-387 (Cuvres 4, 1749-1805).

[20] —, Sur le problème d'analyse relatif à l'équilibre des plaques élastiques encastrées, Mémoire couronné en 1907 par l'Académie: Prix Vaillant, Mémoires présentés par divers savants à l'Académie des Sciences 33, No. 4 (Euvres 2, 515-629).

[21] —, Sur le module maximum d'une fonction et de ses dérivées, Bull. Soc. Math. France 42 (1914), 68-72.

[22] G. H. Hardy, E. Landau and J. E. Littlewood, Some inequalities satisfied by the integrals of derivatives of real or analytic functions, Math. Z. 39 (1935), 677-695.

[23] G. H. Hardy and J. E. Littlewood, Contributions to the arithmetic theory of series, Proc. London Math. Soc. (2) 11 (1912-1913), 411-478.

[24] - - - Some integral inequalities connected with the calculus of variations, Quart. J. Math. 3 (1932), 242-252.

[25] A. Kałamajska, Pointwise multiplicative inequalities and Nirenberg type estimates in weighted Sobolev spaces, Studia Math. 108 (1994), 275-290.

[26] A. Kałamajska and K. Pietruska-Pałuba, Logarithmic version of interpolation inequalities for derivatives, J. London Math. Soc. (2) 70 (2004), 691-702.

[27] - - - Interpolation inequalities for derivatives in Orlicz spaces, Indiana Univ. Math. J., to appear.

[28] -, -, Gagliardo-Nirenberg inequalities in logarithmic spaces, Colloq. Math., to appear.

[29] R. Kerman and A. Torchinsky, Integral inequalities with weights for the Hardy maximal function, Studia Math. 71 (1981/82), 277-284.

[30] H. Kita, Inequalities with weights for maximal functions in Orlicz spaces, Acta Math. Hungar. 72 (1996), 291-305.

[31] - A reverse weighted inequality for the Hardy-Littlewood maximal function in Orlicz spaces, ibid. 98 (2003), 85-101.

[32] A. Kneser, Studien über die Bewegungsvorgänge in der Umgebung instabiler Gleichgewichtslagen, J. Reine Angew. Math. 118 (1897), 186-223. 
[33] V. Kokilashvili and M. Krbec, Weighted Inequalities in Lorentz and Orlicz Spaces, World Sci., Singapore, 1991.

[34] A. N. Kolmogorov, On inequalities between upper bounds of consecutive derivatives of an arbitrary function defined on an infinite interval, Uchen. Zap. MGU Mat. 30 (1939), no. 3, 13-16 (in Russian).

[35] M. A. Krasnosel'skiǔ and Ya. B. Rutickiı̆, Convex Functions and Orlicz Spaces, Noordhoff, Groningen, 1961.

[36] M. Kwong and A. Zettl, Norm inequalities for derivatives, in: Ordinary and Partial Differential Equations (Dundee, 1980), Lecture Notes in Math. 846, Springer, Berlin, 1981, 227-243.

[37] Q. S. Lai, Two weight $\Phi$-inequalities for the Hardy operator, Hardy-Littlewood maximal operator, and fractional integrals, Proc. Amer. Math. Soc. 118 (1993), 129-142.

[38] E. Landau, Einige Ungleichungen für zweimal differentierbare Funktionen, Proc. London Math. Soc. (2) 13 (1914), 43-49.

[39] —, Die Ungleichungen für zweimal differentierbare Funktionen, Meddelelser Kobenhavn 6 (1925), no. 10, 1-49.

[40] -, Über einer Satz von Herrn Esclangon, Math. Ann. 102 (1929), 177-188.

[41] V. G. Maz'ya and T. Shaposhnikova, On pointwise interpolation inequalities for derivatives, Math. Bohemica 124 (1999), 131-148.

[42] -, -, Pointwise interpolation inequalities for derivatives with best constants, Funktsional. Anal. i Prilozhen. 36 (2002), no. 1, 36-58, (Russian); English transl.: Funct. Anal. Appl. 36 (2002), no. 1, 30-48.

[43] D. S. Mitrinović, J. E. Pečarić and A. M. Fink, Inequalities Involving Functions and Their Derivatives, Kluwer, Dordrecht, 1991.

[44] B. Muckenhoupt, Weighted norm inequalities for the Hardy maximal function, Trans. Amer. Math. Soc. 165 (1972), 207-226.

[45] L. Nirenberg, On elliptic partial differential equations, Ann. Scuola Norm. Sup. Pisa 13 (1959), 115-162.

[46] D. Ornstein, A non-inequality for differential operators in the $L_{1}$-norm, Arch. Rat. Mech. Anal. 2 (1962), 40-49.

[47] W. Pompe, Existence theorems in the viscoplasticity theory, Ph.D. thesis, Darmstadt Techn. Univ. Diss. 2003, Shaker Verlag, 2003, Aachen.

[48] E. M. Stein, Note on the class LLog L, Studia Math. 32 (1969), 305-310.

[49] P. Strzelecki, Gagliardo-Nirenberg interpolation inequalities with a BMO term, J. London Math. Soc., to appear.

[50] A. Torchinsky, Real-Variable Methods in Harmonic Analysis, Academic Press, New York, 1986.

[51] H. Tuominen, Orlicz-Sobolev spaces on metric measurable spaces, $\mathrm{PhD}$ thesis, Univ. of Jyväskylä, Helsinki, 2004.

Institute of Mathematics

Warsaw University

Banacha 2

02-097 Warszawa, Poland

E-mail: kalamajs@mimuw.edu.pl

kpp@mimuw.edu.pl 